\title{
CALIDAD DEL SERVICIO EDUCATIVO EN UNA INSTITUCIÓN DE EDUCACIÓN SUPERIOR-IES PRIVADA DE LA CIUDAD DE PEREIRA (COLOMBIA)
}

\author{
QUALITY OF THE EDUCATIONAL SERVICE IN A PRIVATE INSTITUTION \\ OF HIGHER EDUCATION-HEI IN THE PEREIRA CITY (COLOMBIA)
}

\section{Jorge Andrés Arroyave García, * Olga Lucía Hurtado-Cardona}

Docente de la Corporación Universitaria Minuto de Dios -UNIMINUT0-.. jarroyabega@uniminuto.edu.co

Docente asociada de la Fundación Universitaria Comfamiliar Risaralda. ohurtado@uc.edu.co Código ORCID: 0000-0003-0809-8105

RESUMEN

ABSTRACT

Siendo la calidad en el servicio de la Educación Superior un concepto multidimensional que depende del marco específico en el que opere, se pretende determinar la calidad del servicio educativo para los estudiantes de pregrado de una Facultad de Ciencias Administrativas de una Institución de Educación Superior privada ubicada en el Eje Cafetero colombiano. Para lo anterior se utiliza el Modelo Servqual realizando la medición de la calidad percibida a través de una evaluación por separado de las expectativas y las percepciones del servicio esperado y recibido, utilizando un tipo de investigación concluyente descriptivo de carácter formal y estructurado, en el cual, se encontraron deficiencias en la oferta; dichas falencias no están permitiendo óptimos niveles de percepción, evidenciándose en las brechas encontradas. De igual forma el ejercicio permite complementar las herramientas de autorregulación y autoevaluación contempladas y direccionadas por el Ministerio de Educación Nacional.

PALABRAS CLAVE: calidad del servicio, educación superior, modelo servqual, brechas del servicio.

Being the quality of service in Higher Education a multidimensional concept that depends on the specific framework in which it operates, the aim it is establish the quality of the educational service for undergraduate students of a Faculty of Administrative Sciences of a Private Higher Education Institution (HEI) located in the Colombian Coffee Region. For this, the Servqual Model is used, measuring the perceived quality through a separate evaluation of the expectations and perceptions of the expected and received service, using a type of conclusive descriptive research of a formal and structured nature, in which, deficiencies were found in the offer; these shortcomings are not allowing optimal levels of perception, evidencing in the gaps found. In the same way, the exercise complements the tools of self-regulation and self-evaluation contemplated and directed by the Ministry of National Education.

KEYWORDS: Quality of Service, Higher Education, Servqual Model, Service Caps. 


\section{Introducción}

La educación en Colombia está reglamentada por la Ley 30 de 1992 y en ella se establecen los parámetros a través de los cuales ésta se debe desarrollar; dicha ley tiene como propósito que el servicio público de la educación se ejerza con calidad, y por tanto, el Ministerio de Educación Nacional (MEN) ha fijado unos estándares que tienen la intención de garantizar el debido seguimiento y cumplimiento por parte de las IES. Teniendo claro que la valoración de la calidad de un servicio -como el educativono solamente se pueda dar o medir por el cumplimiento de las normas, se debe efectuar una valoración directa en los actores principales que son los estudiantes, ya que, por la característica de intangibilidad, la medición se debe establecer desde parámetros de percepción de acuerdo a lo recibido frente a lo esperado.

La calidad en la perspectiva del servicio, según Lewis y Mitchel (1990), debe estar enfocada en la satisfacción de las necesidades y las expectativas de los clientes. De igual forma, la medición de la calidad de los servicios se realiza a través del desempeño que se ha obtenido por la relación entre las expectativas previas y las percepciones posteriores (Parasuraman et al., 1985; Lewis \& Mitchell, 1990), procedimientos que han sido utilizados en universidades públicas, como las del este de África (Cheruityot \& Maru, 2013).

Existen dos grandes corrientes para la modelización de la calidad de los servicios; la primera de ellas es la escuela norte-europea liderada por Grönroos (1984), quien afirma que la calidad percibida por los clientes se fundamenta en tres aspectos: la calidad técnica, la calidad funcional y la imagen de la organización; la segunda escuela es la norteamericana liderada por Parasuraman, quien -en conjunto con otros- desarrolla la teoría de brechas en el servicio a finales del siglo XX, y desde la cual crean el modelo de medición de la calidad en los servicios llamado Servqual (Ríos \& Santomá, 2008). El presente estudio basa su perspectiva en este modelo, el cual utiliza un cuestionario que consta de cinco dimensiones: 1) Elementos tangibles, 2) Confiabilidad, 3) Capacidad de respuesta, 4) Garantía y 5) Empatía.

Para efectuar la investigación se eligió una IES de carácter privado en la ciudad de Pereira -ubicada en el Eje Cafetero Colombiano-y, de manera específica, a los estudiantes de los programas de pregrado que conforman la Facultad de Ciencias Administrativas, cuyo propósito fue establecer las expectativas presentes al momento de iniciar la formación académica y las percepciones generadas al culminar sus respectivas carreras profesionales. Para efectuar la medición objeto de estudio se aplicó el Modelo Servqual, pertinente para evaluar la calidad en los servicios pres- tados por una organización (Parasuraman et al, 1985), y en donde se puede dar cuenta de los procesos que están teniendo falencias para eliminarlos o evitarlos, consiguiendo la recompensa que los alumnos desean, y por tanto, favorecer los márgenes de rentabilidad y participación de la IES en el mercado.

Para la aplicación del cuestionario Servqual, las expectativas se midieron a través de 22 preguntas en una escala de siete puntos con las categorías de respuesta que van desde "nada esencial" hasta "absolutamente esencial”. Del mismo modo, las percepciones -también con 22 preguntas-, se calcularon con otra escala de siete puntos cuyas opciones de respuesta van desde "totalmente de acuerdo" hasta "totalmente en desacuerdo". Por tanto, siguiendo a Zeithaml (2009), Servqual se convierte en una escala de 44 reactivos que mide las expectativas frente a las percepciones de los estudiantes respecto a las cinco dimensiones de calidad en el servicio mencionadas.

Dentro de la metodología usada para la aplicación del estudio se recurre a las investigaciones de tipo Concluyente-Descriptivo, y así someter los datos a un análisis cuantitativo con el que se llega a tomas de decisiones administrativas (Hernández, 2014), teniendo en cuenta el carácter finito de la población y una técnica de muestreo probabilística aleatoria simple. De esta manera, y para que se cumpla el objetivo de la medición a través del Modelo Servqual, los encuestados manifiestan su nivel de expectativas y de percepciones, de modo que se pueda llegar a comprender la satisfacción que existe alrededor de la calidad del servicio educativo brindado; posteriormente, se identifican y compararan las brechas obtenidas en las cinco dimensiones de calidad en el servicio educativo.

Lo anterior permite que las cinco dimensiones sean ubicadas en mapas de posicionamiento y de umbral de satisfacción dentro de un plano cartesiano (Martínez, 2012) procediendo a concluir sobre el diagnóstico encontrado, dando así respuesta al objetivo de investigación en cuanto a establecer las brechas existentes entre las expectativas y las percepciones de la calidad del servicio educativo por parte de los estudiantes de una Facultad de Ciencias Administrativas en una IES privada de la ciudad de Pereira.

\section{Metodología}

Teniendo en cuenta que se busca establecer las expectativas y percepciones en cuanto a la calidad del servicio educativo en estudiantes de primer y último año, respectivamente, de una Facultad de Ciencias Administrativas en una IES de la ciudad de Pereira, se presenta ontológicamente una mirada desde los hechos sociales para así poder realizar una interpretación de una realidad que, desde 
la epistemología según Thiétart (1999), busca la comprensión de la misma de acuerdo a un tiempo y a un espacio determinados. Desde la lógica de razonamiento, se recurre a un enfoque de abducción, ya que el interés se centra en la elaboración de inferencias combinando teoría con realidad (Bryman, 2012).

El tipo de investigación utilizado es el concluyente-descriptivo -de carácter formal y estructurado- cuyo objetivo es describir características y fenómenos de un mercado, además de probar hipótesis específicas y examinar relaciones particulares (Hernández, Fernández \& Baptista, 2014). Este diseño de investigación se basa en tamaños de muestra grandes y representativos de una población detallada, sometiendo los datos a un análisis cuantitativo con los que se llega con toda confianza a la posibilidad de tomar decisiones administrativas (Malhotra, 2008).

La técnica de muestreo es de carácter Probabilístico (Aleatoria Simple), propia de investigaciones concluyentes para elegir a quienes hacen parte del estudio, pues cada uno de los elementos muestrales cuenta con la misma probabilidad de ser seleccionado para intervenir en el proceso de recolección de datos, obedeciendo esto a una fuente primaria de información (Malhotra, 2008).

Respecto a la técnica de recolección de la información, Ravichandran et al (2010) afirman que la percepción del consumidor en cuanto a la calidad del servicio es un proceso complejo del cual han sugerido múltiples dimensiones, siendo el Modelo Servqual uno de los más populares. Parasuraman et al (1985) desarrollaron este modelo y lo utilizaron en la comercialización de servicios, basándose en la brecha de percepción entre la calidad esperada del servicio y el servicio recibido a través de cinco dimensiones, que inicialmente fueron 10.

Para el presente caso de aplicación, se implementa -con la respectiva adaptación- el cuestionario del Modelo Servqual, muy pertinente para evaluar la calidad en los servicios prestados por una organización, en donde ésta puede darse cuenta de los procesos que están teniendo fortalezas para seguirlas afianzando, o de las falencias para eliminarlas o evitarlas, consiguiendo la recompensa de sus clientes y, por tanto, una mayor rentabilidad y participación en el mercado. Dicho cuestionario consta de las siguientes cinco dimensiones: 1) Elementos tangibles (apariencia de los materiales físicos); 2) Confiabilidad (fiable, desempeño preciso); 3 ) Capacidad de respuesta (prontitud, utilidad); 4) Garantía (pericia, cortesía, credibilidad y certidumbre); y 5) Empatía (fácil acceso, buena comunicación y comprensión del usuario).

De esta manera, y para que se cumpla el objetivo de la medición a través del Modelo Servqual, los encuestados refieren su nivel de expectativa y de percepción en cada una de las cinco dimensiones, de modo que se puede llegar a comprender la satisfacción que existe alrededor de la calidad del servicio educativo brindado. Es por esto que los estudiantes de primer año de pregrado responden todo lo relacionado con las expectativas, ya que por estar iniciando sus programas académicos presentan un ideal de calidad en el servicio educativo; mientras que, aquellos que se encuentran en el último año han utilizado por más tiempo el servicio, pudiendo evaluar el desempeño del mismo; finalmente, se realizan las comparaciones respectivas y el análisis de las brechas encontradas en cada una de las dimensiones mencionadas (expectativas vs percepciones).

Las expectativas de los usuarios, a través de 22 preguntas, se miden en una escala de siete puntos con las categorías de respuesta que van desde "nada esencial" hasta "absolutamente esencial". Del mismo modo, las percepciones, que también poseen 22 preguntas, se evalúan con otra escala de siete puntos cuyas opciones de respuesta van desde "totalmente de acuerdo" hasta "totalmente en desacuerdo". Siendo así, Servqual se convierte en una escala de 44 reactivos que mide las expectativas vs las percepciones de los usuarios respecto a las cinco dimensiones de calidad en el servicio (Parasuraman et al, 1985).

Una vez se efectúa el diagnóstico de las expectativas y las percepciones por medio de la aplicación de los respectivos cuestionarios pertenecientes al Modelo, se identifican y comparan las brechas obtenidas en las cinco dimensiones de calidad en el servicio educativo. Ello permite realizar mapas de posicionamiento y de umbral de satisfacción (Martínez, 2012) con el propósito de visualizar y analizar estratégicamente los resultados.

\section{Resultados}

Para una mayor comprensión, se presentan los resultados con base en los siguientes aspectos: a) Población y tamaño de la muestra; b) Las cinco dimensiones del servicio; c) Los puntajes ponderados, d) El posicionamiento de las dimensiones en los cuatro cuadrantes, e) El posicionamiento de las dimensiones en el umbral de satisfacción y, e) La comprobación de la existencia del problema.

\section{a)Población y tamaño de la muestra:}

Para la obtención de los resultados, se tuvo en cuenta una población de carácter finito ubicada en un lugar específico, es decir, la institución educativa. Se utilizó una fórmula estadística en donde el nivel de confianza es del $95,5 \%$ y el margen de error se determinó en un $4 \%$ con base en la variable satisfacción con el servicio. Siendo así, para el cálculo del 
tamaño de la muestra, se estableció que los estudiantes de primer año son 207 y la muestra que se decidió fue de 156 estudiantes; de igual forma, los estudiantes de último año son 199 en donde la muestra es de 152 estudiantes, tal y como se muestra a continuación:

$$
\mathrm{n}=\frac{\mathrm{Z}^{2} \mathrm{P}(1-\mathrm{P}) \mathrm{N}}{\mathrm{me}^{2}(\mathrm{~N}-1)+\mathrm{Z}^{2} \mathrm{P}(1-\mathrm{P})}
$$

Donde:

n: tamaño de la muestra

$\mathrm{Z}$ : confiabilidad

me: margen de error

$\mathrm{N}$ :tamaño de la población

P: probabilidad de éxito

$$
\mathrm{n}=\frac{\left(1,96^{2}\right)(0,5)(0,5)(207)}{(0,04)^{2}(206)+(1,96)^{2}(0,5)(0,5)}
$$

Donde:

$Z=1,96 \rightarrow$ confiabilidad del $95,5 \%$

$\mathrm{P}=$ probabilidad de satisfacción, de acuerdo a expectativa y percepción.

$\mathrm{me}=4 \%$ margen de error permitido en el estudio, de acuerdo a la variable satisfacción del servicio.

El tamaño de la muestra para los estudiantes de primer año, de acuerdo a la aplican=154,11 ción de la fórmula estadís tica, es de 154; no obstante, los investigadores tomaron de muestra a 156 de ellos.
Ahora:

$$
\mathrm{n}=\frac{\left(1,96^{2}\right)(0,5)(0,5)(199)}{(0,04)^{2}(198)+(1,96)^{2}(0,5)(0,5)}
$$

Donde:

$\mathrm{Z}=1,96$ - confiabilidad del 95,5\%

$\mathrm{P}=$ probabilidad de satisfacción, de acuerdo a expectativa y percepción.

$\mathrm{me}=4 \%$ margen de error permitido en el estudio, de acuerdo a la variable satisfacción del servicio.

El tamaño de la muestra para los estudiantes de último año, de acuerdo a la aplicación de la n=149,64 fórmula estadística, es de 150; sin embargo, los investigadores tomaron de muestra a 152 de ellos.

\section{b)Las cinco dimensiones del servicio:}

Las tablas 1, 2, 3, 4 y 5 muestran el consolidado de resultados en cada dimensión del servicio evaluada, las cuales contienen además el tamaño de brecha (positiva o negativa) resultante

\begin{tabular}{|c|c|c|c|c|}
\hline EXPECTATIVAS & PUNTAJE & PERCEPCIONES & PUNTAJE & BRECHA \\
\hline $\begin{array}{l}\text { Expectativas promedio para } \\
\text { Aspectos Físicos o Tangibles }\end{array}$ & 6,04 & $\begin{array}{l}\text { Percepciones promedio para } \\
\text { Aspectos Físicos o Tangibles }\end{array}$ & 5,52 & $-0,52$ \\
\hline $\begin{array}{l}\text { Las IES excelentes cuentan con } \\
\text { salones, auditorios, cafeterías, } \\
\text { áreas de esparcimiento, etc., en } \\
\text { óptimas condiciones para ejercer la } \\
\text { actividad educativa. }\end{array}$ & 5,95 & $\begin{array}{l}\text { La IES a la cual pertenece cuenta con } \\
\text { salones, auditorios, cafeterías, } \\
\text { áreas de esparcimiento, etc, en } \\
\text { óptimas condiciones para que usted } \\
\text { ejerza su actividad educativa. }\end{array}$ & 5,18 & $-0,77$ \\
\hline $\begin{array}{l}\text { Las instalaciones físicas de las IES } \\
\text { excelentes son visualmente } \\
\text { atractivas. }\end{array}$ & 5,78 & $\begin{array}{l}\text { Las instalaciones físicas de la IES a } \\
\text { la cual pertenece le son visualmente } \\
\text { atractivas. }\end{array}$ & 5,60 & $-0,18$ \\
\hline $\begin{array}{l}\text { Al llegar a una IES excelente, se } \\
\text { espera encontrar a los docentes y } \\
\text { administrativos con una } \\
\text { presentación impecable. }\end{array}$ & 5,94 & $\begin{array}{l}\text { Considera que los docentes y } \\
\text { administrativos de la IES a la cual } \\
\text { pertenece tienen un aspecto pulcro y } \\
\text { son bien presentados. }\end{array}$ & 5,80 & $-0,15$ \\
\hline $\begin{array}{l}\text { Considera que la iluminación no } \\
\text { solamente es necesaria para una } \\
\text { buena educación, sino que también } \\
\text { le cautiva para utilizar más } \\
\text { servicios. }\end{array}$ & 6,00 & $\begin{array}{l}\text { Considera que la iluminación de la } \\
\text { IES a la cual pertenece es adecuada y } \\
\text { estimula su hábito de estudiar. }\end{array}$ & 5,70 & $-0,30$ \\
\hline $\begin{array}{l}\text { Quisiera llegar a una IES y ubicarse } \\
\text { fácilmente con la señalización } \\
\text { existente. }\end{array}$ & 6,04 & $\begin{array}{l}\text { La IES a la cual pertenece cuenta con } \\
\text { la señalización necesaria para su } \\
\text { adecuada ubicación al interior de la } \\
\text { misma. }\end{array}$ & 5,43 & $-0,61$ \\
\hline $\begin{array}{l}\text { El acceso a una IES excelente debería } \\
\text { tener un buen espacio de circulación } \\
\text { que facilite el ingreso y salida de los } \\
\text { estudiantes. }\end{array}$ & 6,15 & $\begin{array}{l}\text { Para usted, el acceso a la IES a la } \\
\text { cual pertenece, posee un buen } \\
\text { espacio de circulación que facilita el } \\
\text { ingreso y salida de los estudiantes. }\end{array}$ & 5,21 & $-0,94$ \\
\hline $\begin{array}{l}\text { Como estudiante de una IES, } \\
\text { esperaría encontrar los espacios } \\
\text { aseados y con una apariencia } \\
\text { agradable. }\end{array}$ & 6,44 & $\begin{array}{l}\text { Como estudiante de la IES a la cual } \\
\text { pertenece encuentra espacios } \\
\text { aseados y con una apariencia } \\
\text { agradable. }\end{array}$ & 5,74 & $-0,69$ \\
\hline
\end{tabular}
de restar las percepciones de las expectativas.

Tabla 1Consolidado de Resultados. Dimensión: Aspectos Físicos o Tangibles 
En la dimensión de aspectos físicos o tangibles se puede observar una brecha negativa de -0,52, lo que indica que la IES no está cumpliendo a cabalidad con las expectativas generadas en los estudiantes; esto se evidencia especialmente en tres aspectos: salones, auditorios, espacios de esparcimiento y en las vías de acceso a la Institución; sin embargo, no se puede dejar de lado que siendo importantes el aseo y la apariencia física, están siendo descuidados.

Tabla 2 Consolidado de Resultados. Dimensión: Confiabilidad

\begin{tabular}{lclc}
\hline \multicolumn{1}{c}{ EXPECTATIVAS } & PUNTAJE & \multicolumn{1}{c}{ PERCEPCIONES } & PUNTAJE \\
\hline $\begin{array}{l}\text { Expectativas promedio para } \\
\text { Confiabilidad }\end{array}$ & 5,96 & $\begin{array}{l}\text { Percepciones promedio para } \\
\text { Confiabilidad }\end{array}$ & 5,35 \\
\hline $\begin{array}{l}\text { Cuando las IES excelentes prometen } \\
\text { hacer algo en un momento } \\
\text { determinado, lo cumplen. }\end{array}$ & 5,98 & $\begin{array}{l}\text { La IES a la cual pertenece le entrega } \\
\text { el servicio educativo conforme a lo } \\
\text { prometido. }\end{array}$ & 5,39 \\
\hline $\begin{array}{l}\text { Cuando los estudiantes tienen un } \\
\text { problema, las IES excelentes } \\
\text { demuestran un sincero interés en } \\
\text { resolverlo. }\end{array}$ & 5,94 & $\begin{array}{l}\text { La IES a la cual pertenece le muestra } \\
\text { problemas o inquietudes que se le } \\
\text { han presentado al interior de sus } \\
\text { instalaciones. }\end{array}$ & -0,59 \\
\hline $\begin{array}{l}\text { Las IES excelentes realizan bien el } \\
\text { servicio desde el primer momento. }\end{array}$ & 5,96 & $\begin{array}{l}\text { La IES a la cual pertenece le ha } \\
\text { brindado bien el servicio desde el } \\
\text { primer momento. }\end{array}$ & 5,23 \\
\hline
\end{tabular}

La confiabilidad presenta una brecha negativa de -o,62 -mayor que la dimensión de aspectos físicos- haciendo que sea preocupante por tratarse del cumplimiento de la promesa básica del servicio educativo, la solución de problemas al interior de la IES y, la prontitud y eficiencia de los servicios ofertados.

Tabla 3 Consolidado de Resultados. Dimensión: Capacidad de Respuesta

\begin{tabular}{|c|c|c|c|c|}
\hline EXPECTATIVAS & PUNTAJE & PERCEPCIONES & PUNTAJE & BRECHA \\
\hline $\begin{array}{l}\text { Expectativas promedio para } \\
\text { Capacidad de Respuesta }\end{array}$ & 5,94 & $\begin{array}{l}\text { Percepciones promedio para } \\
\text { Capacidad de Respuesta }\end{array}$ & 5,45 & $-0,49$ \\
\hline $\begin{array}{l}\text { Los docentes y administrativos de } \\
\text { las IES excelentes dirán a los } \\
\text { estudiantes exactamente en cuánto } \\
\text { tiempo serán prestados los servicios } \\
\text { educativos. }\end{array}$ & 5,90 & $\begin{array}{l}\text { Los docentes y administrativos de la } \\
\text { IES a la cual pertenece le han dicho } \\
\text { exactamente en cuánto tiempo } \\
\text { serán prestados los servicios } \\
\text { educativos. }\end{array}$ & 5,49 & $-0,41$ \\
\hline $\begin{array}{l}\text { Los docentes y administrativos de } \\
\text { las IES excelentes darán un servicio } \\
\text { rápido a los estudiantes. }\end{array}$ & 5,94 & $\begin{array}{l}\text { Los docentes y administrativos de la } \\
\text { IES a la cual pertenece le han } \\
\text { proporcionado un servicio rápido. }\end{array}$ & 5,42 & $-0,52$ \\
\hline $\begin{array}{l}\text { Los docentes y administrativos de } \\
\text { las IES excelentes estarán siempre } \\
\text { dispuestos a ayudar a los } \\
\text { estudiantes. }\end{array}$ & 6,06 & $\begin{array}{l}\text { Los docentes y administrativos de la } \\
\text { IES a la cual pertenece han estado } \\
\text { siempre dispuestos a ayudarle. }\end{array}$ & 5,61 & $-0,45$ \\
\hline $\begin{array}{l}\text { Los docentes y administrativos de } \\
\text { las IES excelentes nunca estarán } \\
\text { demasiado ocupados para responder } \\
\text { a las peticiones de los estudiantes. }\end{array}$ & 5,85 & $\begin{array}{l}\text { Los docentes y administrativos de la } \\
\text { IES a la cual pertenece nunca están } \\
\text { demasiado ocupados para responder } \\
\text { a sus peticiones y solicitudes. }\end{array}$ & 5,29 & $-0,56$ \\
\hline
\end{tabular}

Fuente: Elaboración Propia

La capacidad de respuesta presenta una brecha negativa de -o,49, indicando que a los docentes y administrativos de la IES les falta compromiso en la prestación del servicio educativo y en los aspectos que giran alrededor de éste como lo son: el tiempo de entrega, la rapidez de la oferta, la disposición de ayuda $y$, la voluntad de respuesta a peticiones y solicitudes; de igual forma, se evidencia la carencia de acciones generadoras de sentido de pertenencia para quienes hacen parte de la institución y que son pilar fundamental del servicio ofertado. 
Tabla 4 Consolidado de Resultados. Dimensión: Garantía

\begin{tabular}{|c|c|c|c|c|}
\hline EXPECTATIVAS & PUNTAJE & PERCEPCIONES & PUNTAJE & BRECHA \\
\hline $\begin{array}{l}\text { Expectativas promedio para } \\
\text { Garantía }\end{array}$ & 6,20 & $\begin{array}{l}\text { Percepciones promedio para } \\
\text { Garantía }\end{array}$ & 5,63 & $-0,58$ \\
\hline $\begin{array}{l}\text { Los docentes y administrativos de } \\
\text { las IES excelentes tienen } \\
\text { constantemente una actitud cortés } \\
\text { hacia los estudiantes. }\end{array}$ & 6,08 & $\begin{array}{l}\text { Los docentes y administrativos de la } \\
\text { IES a la cual pertenece han tenido } \\
\text { constantemente una actitud cortés } \\
\text { con usted. }\end{array}$ & 5,61 & $-0,47$ \\
\hline
\end{tabular}

En la dimensión de garantía, la cual tiene como enfoque el análisis de las condiciones y capacidades que poseen los docentes y los administrativos de la Institución para asegurar la oferta del servicio, se encuentra una brecha negativa de $-0,58$, lo cual genera inquietudes relacionadas con los procesos de formación y ca- pacitación brindados a los colaboradores con el propósito de elevar los niveles de satisfacción y las valoraciones realizadas en este aspecto; es decir, se debe buscar que la cadena de utilidad del servicio fluya positivamente desde quienes ofertan al servicio hasta quienes lo reciben.

Tabla 5 Consolidado de Resultados. Dimensión: Empatía

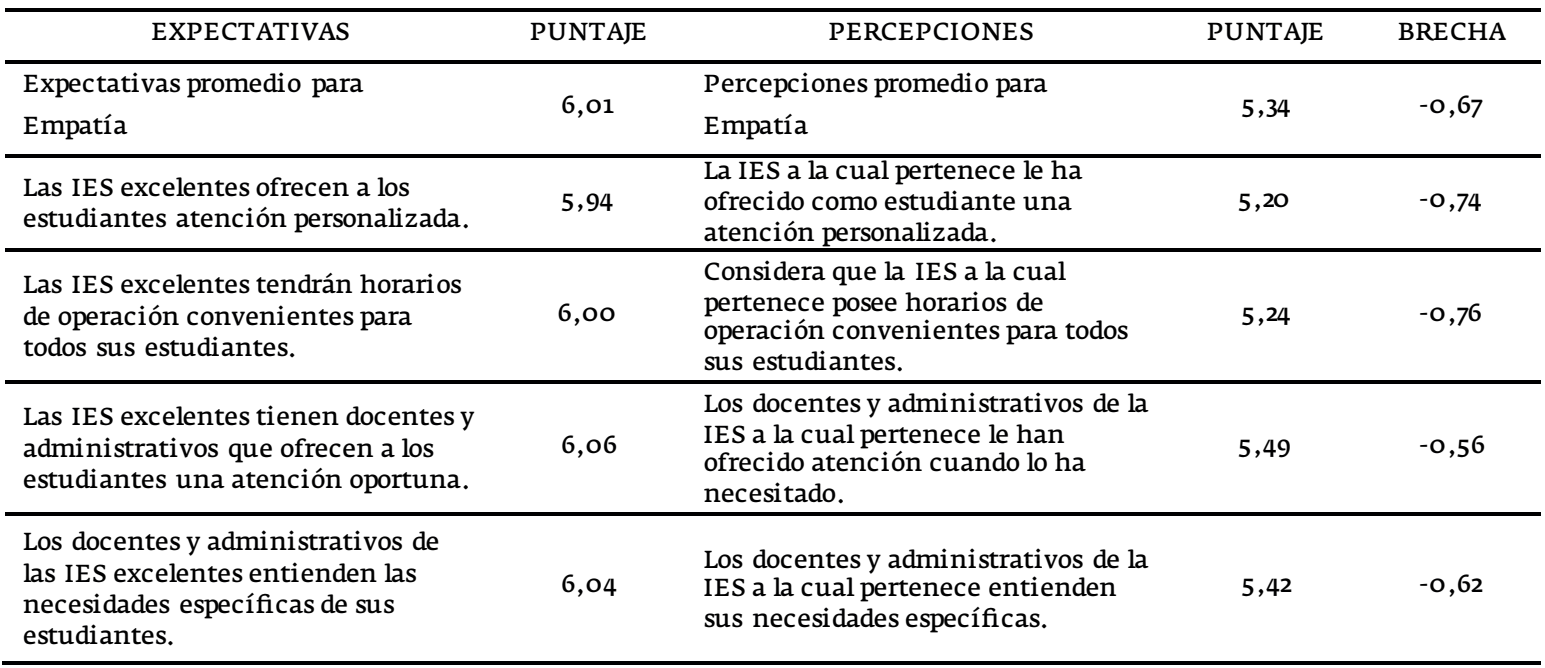

Al comprender la dimensión de empatía como aquella capacidad que posee la IES de ponerse en el lugar de los usuarios de los servicios educativos y, de esta manera, brindar una oferta bajo las necesidades y requerimientos de los estudiantes, se observa una brecha negativa de $-0,67$, entendiendo que las condiciones bajo las cuales se entrega la oferta están dadas en mayor medida para la comodidad de los profesores y administrativos, y no tanto de los estudiantes.

Como puede observarse en todos los casos, en las cinco dimensiones alusivas a la calidad del servicio educativo, las percepciones son evaluadas de manera inferior a las expectativas, es decir que, lo ideal o esperado es mucho mayor a lo recibido realmente, conllevando a unos resultados de brecha negativos.

\section{c)Los Puntajes Ponderados:}

Siguiendo a Martínez (2012), debido a que cada una de las cinco dimensiones no necesariamente posee la misma importancia en el momento de evaluar la calidad del servicio, se 
debe realizar un ejercicio de ponderación distribuyendo cien (100) puntos entre ellas. Dicha ponderación fue efectuada teniendo en cuenta las normas estipuladas en el Decreto 1295 de 2010 del MEN (compilado en el Decreto Único Reglamentario del Sector Educación 1075 de 2015), el cual regula la oferta y desarrollo de programas académicos de educación superior, en donde a través de quince (15) condiciones de calidad se establecen los parámetros bajo los cuales las IES deben brindar sus servicios de formación.

En este sentido, los investigadores otorgaron diferentes pesos a cada dimensión del servicio con el fin de efectuar en el procesamiento de datos, una etapa consistente en ajustar los valores de acuerdo a la ponderación realizada. Es así como se recalculan los valores de las expectativas y las percepciones para cada una de las cinco dimensiones, multiplicando los originales por el peso asignado a cada una de ellas; y una vez ejecutado esto, se vuelve a calcular el tamaño de brecha de la misma manera como se hizo en el consolidado de resultados (restando la percepción de la expectativa), pero en esta ocasión tomando los Puntajes Ponderados. Seguidamente se presentan los resultados de esta fase del procesamiento de datos en la Tabla 6:

Tabla 6 Puntajes Ponderados

\begin{tabular}{|c|c|c|c|c|c|c|c|c|}
\hline \multicolumn{2}{|c|}{ Dimensiones SERVQUAL } & \multirow{2}{*}{$\begin{array}{c}\begin{array}{c}\text { Pondera- } \\
\text { ción }\end{array} \\
\text { Peso }\end{array}$} & \multicolumn{3}{|c|}{$\begin{array}{c}\text { Puntajes Obtenidos en Consolidado de } \\
\text { Resultados }\end{array}$} & \multicolumn{3}{|c|}{ Puntajes Obtenidos con Ponderación } \\
\hline Dimensión & Descripción & & Expectativas & Percepciones & Brecha & Expectativas & Percepciones & Brecha \\
\hline $\begin{array}{l}\text { Aspectos } \\
\text { Físicos o } \\
\text { Tangibles }\end{array}$ & $\begin{array}{c}\text { La apariencia } \\
\text { de las } \\
\text { instalaciones, } \\
\text { equipamiento, } \\
\text { personal y } \\
\text { material de } \\
\text { comunicación. }\end{array}$ & 15,00 & 6,04 & 5,52 & $-0,52$ & 90,63 & 82,82 & $-7,81$ \\
\hline $\begin{array}{l}\text { Confiabili- } \\
\text { dad }\end{array}$ & $\begin{array}{l}\text { La aptitud para } \\
\text { entregar el } \\
\text { servicio } \\
\text { prometido de } \\
\text { forma segura, } \\
\text { confiable y } \\
\text { precisa. }\end{array}$ & 30,00 & 5,96 & 5,35 & $-0,61$ & 178,78 & 160,53 & $-18,26$ \\
\hline $\begin{array}{l}\text { Capacidad de } \\
\text { Respuesta }\end{array}$ & $\begin{array}{c}\text { La } \\
\text { predisposición } \\
\text { y rapidez para } \\
\text { ayudar a sus } \\
\text { usuarios. }\end{array}$ & 20,00 & 5,94 & 5,45 & $-0,49$ & 118,78 & 109,08 & $-9,70$ \\
\hline Garantía & $\begin{array}{c}\mathrm{El} \\
\text { conocimiento, } \\
\text { cortesía y } \\
\text { aptitud para } \\
\text { transmitir } \\
\text { confianza y } \\
\text { seguridad. } \\
\end{array}$ & 20,00 & 6,20 & 5,63 & $-0,58$ & 124,01 & 112,50 & $-11,51$ \\
\hline Empatía & & 100,0 & & \multicolumn{4}{|c|}{ Indicador de Nivel de Calidad del Servicio } & $-11,47$ \\
\hline
\end{tabular}

El Indicador de Nivel de Calidad del Servicio $(-11,47)$ surge como resultado de recalcular el promedio de las brechas ponderadas para cada una de las dimensiones -que en este caso es negativo- señalando que las expectativas de los estudiantes no están siendo cubier- tas por las percepciones que ellos tienen del servicio educativo brindado. A medida que el Indicador se acerca a cero (viniendo de un valor negativo), se interpreta que la percepción que se tiene del servicio se va acercando a las expectativas que los estudiantes poseen. 


\section{d)El Posicionamiento de las Dimen- siones en los Cuatro Cuadrantes:}

Los siguientes resultados consisten en la ubicación de las cinco dimensiones del servicio en un plano cartesiano, cuyos cuadrantes son: Tiempo Perdido, Disgustos Menores, Fortalezas y Oportunidades de Mejora. Lo ante- rior, permite identificar claramente los aspectos en los cuales se deben enfocar los esfuerzos para aumentar las percepciones de los usuarios, considerando además que los valores obtenidos ya están ponderados. La figura 1 muestra la posición que ocupa cada dimensión:

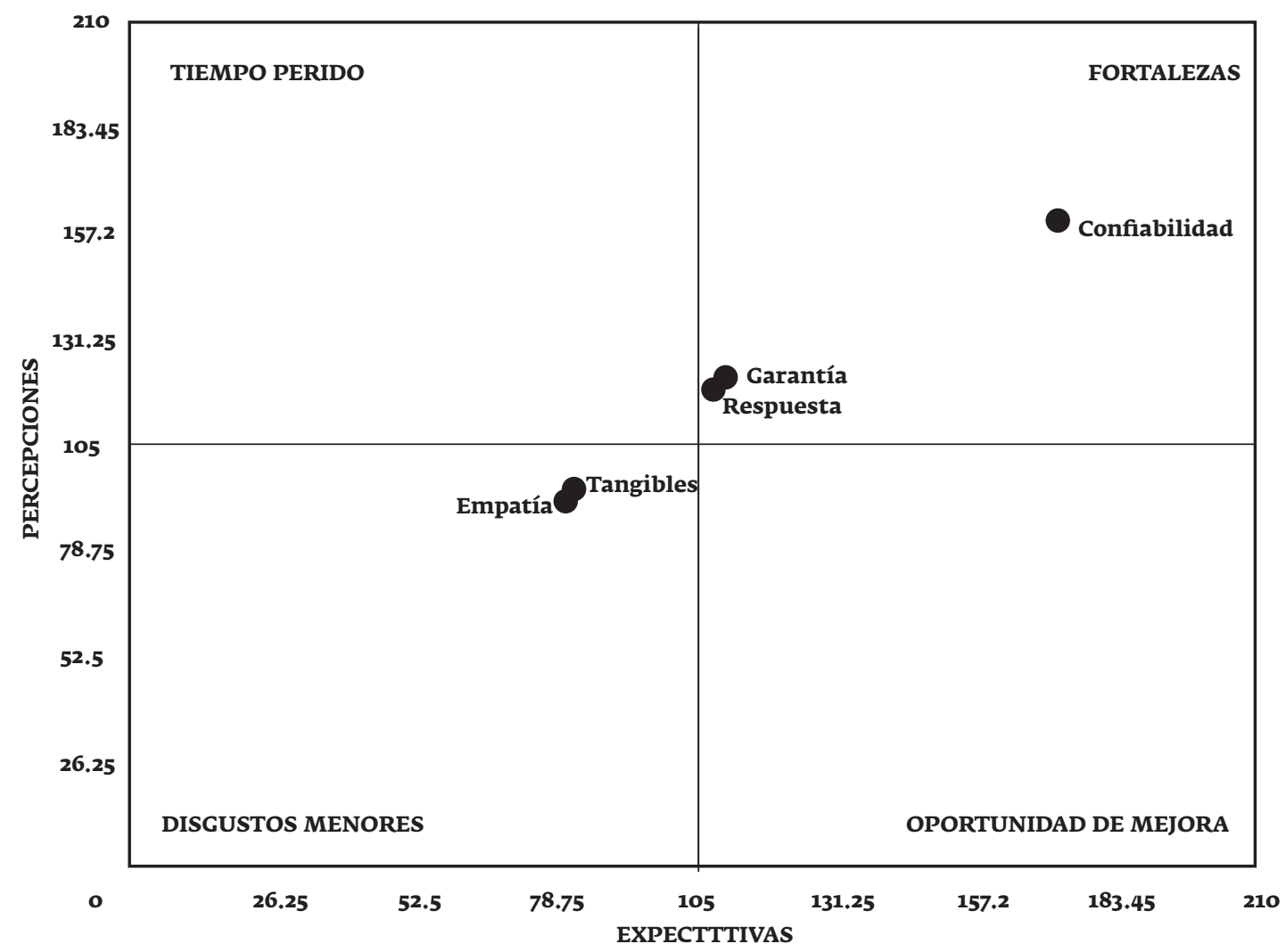

Figura 1 Posicionamiento de las Dimensiones en los Cuatro Cuadrantes

Se interpreta de la figura 1 que a pesar de existir resultados negativos en las brechas, los estudiantes reconocen como fortalezas de la institución las dimensiones relacionadas con la Confiabilidad, la Garantía y la Capacidad de Respuesta. Adicionalmente, los resultados arrojan que las dimensiones de Aspectos Tangibles y Empatía se encuentran en el cuadrante de Disgustos Menores, es decir que, los estudiantes reclaman unas mejores condiciones en las instalaciones y locaciones de la institución, así como un mayor entendimiento a sus necesidades.

\section{e)El Posicionamiento de las Dimensiones en el Umbral de Satisfacción:}

Estos resultados muestran lo cerca o lejos que se encuentra la institución para superar o no, las expectativas de los estudiantes res- pecto al nivel de calidad del servicio educativo percibido. Aquellas dimensiones que se hallan debajo del umbral de satisfacción (ubicado en el plano cartesiano a través de una línea recta punteada que nace en la intersección de los ejes X y Y), representan aspectos del servicio que están por debajo de las expectativas de los estudiantes, mientras que las dimensiones ubicadas sobre dicho umbral, presentan aspectos del servicio educativo que superan las expectativas. Es de anotar que, en estos resultados también se utilizan los valores ajustados por la ponderación dada a cada una de las dimensiones. Lo anterior se muestra en la figura 2: 


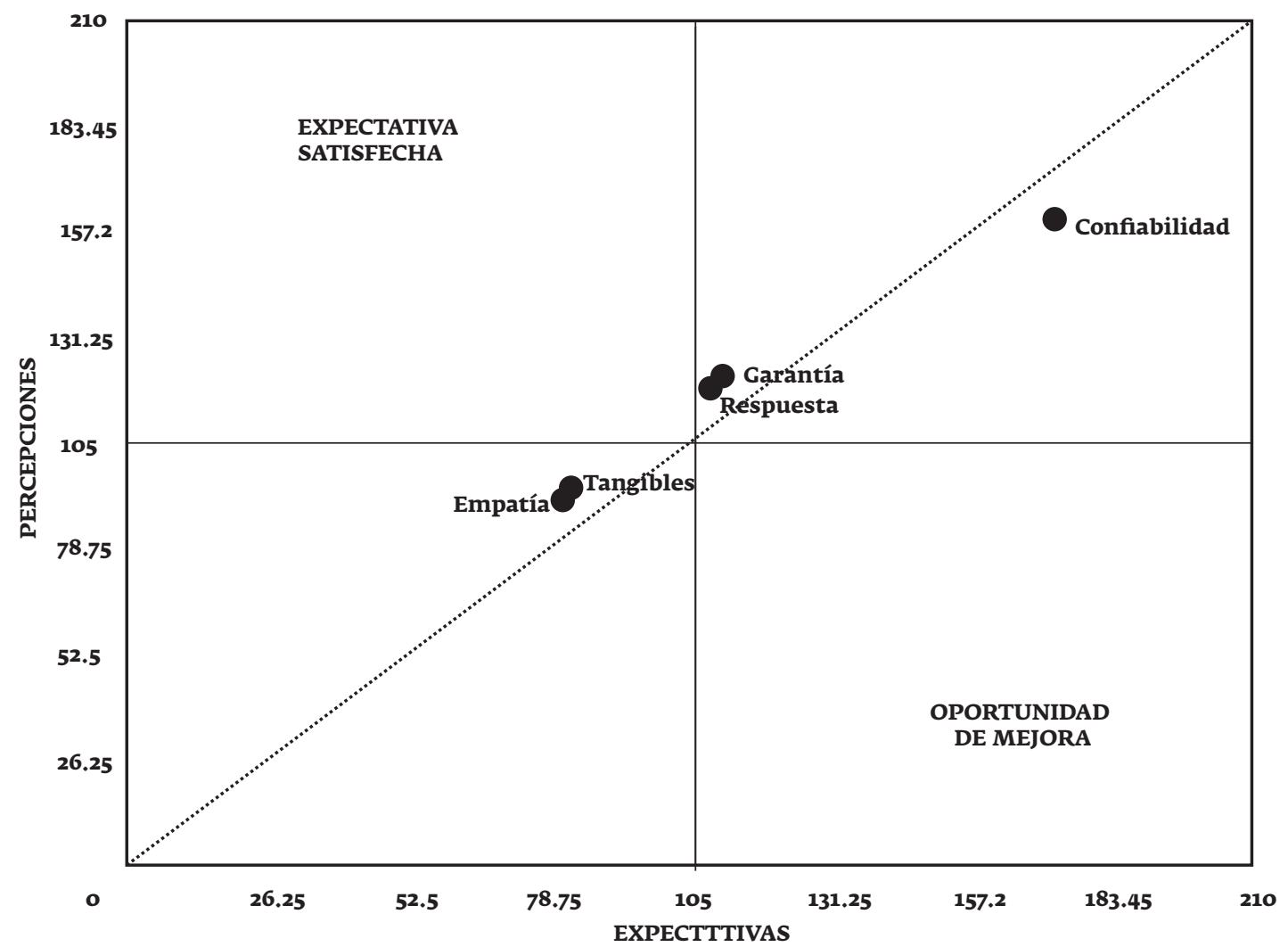

Figura 2 Posicionamiento de las Dimensiones en el Umbral de Satisfacción

Puede verse que de las cinco dimensiones de la calidad del servicio educativo evaluadas por los estudiantes, una se halla por debajo de la línea que delimita el umbral de satisfacción (Confiabilidad), es decir, que se deben implementar de manera prioritaria oportunidades de mejora que permitan acercar las percepciones a las expectativas en este aspecto. Sin embargo, es de resaltar que a pesar de que las otras cuatro dimensiones se encuentran por encima del umbral de satisfacción, éstas están muy cercanas a la línea recta que lo delimita, indicando que es importante que la IES implemente estrategias de sostenimiento de las percepciones y realizar todo lo pertinente para alejarlas positivamente del umbral.

\section{f)Comprobación de la existencia real del Problema:}

Luego de obtener los resultados en el proceso investigativo, en donde las cinco dimensiones evaluadas por los estudiantes de primer y último año, pertenecientes a una Facultad de Ciencias Administrativas de una IES de la ciudad de Pereira, arrojaron cifras negativas, es decir, lo percibido es inferior a lo esperado, se comprueba la existencia del problema, relacionado con la calidad del servicio educativo ofertado por la Institución, la cual, a pesar de implementar continuamente herramientas de autorregulación y autoevaluación contempladas y direccionadas por el Ministerio de Educación Nacional, requiere de procesos en donde el Marketing se convierta en un efectivo instrumento de medición acerca de la satisfacción que los estudiantes deberían poseer del servicio recibido.

De la misma manera, la investigación permite verificar que al aplicar el Modelo Servqual adecuado a una realidad universitaria, se logra establecer a través del proceso de Puntajes Ponderados, el Indicador de nivel de la Calidad del Servicio, el cual es crucial para demostrar en el presente caso que las expectativas de los estudiantes no están siendo satisfechas o cubiertas por las percepciones que ellos tienen del servicio educativo ofertado por la Institución. Incluso, al elaborar los mapas de posicionamiento donde se ubican las dimensiones en los Cuatro Cuadrantes y en el Umbral de Satisfacción, se encontró que efectivamente existen dimensiones situadas en "disgustos menores", las cuales requieren estrategias de mejoramiento prioritarias; y otras, aún situadas en "fortalezas", y superando el umbral de satisfacción, la Institución debe realizar estrategias para incrementar los niveles de satisfacción.

Es debido a lo anterior que se corrobora puntualmente la presencia real de una necesidad relacionada con el mejoramiento imperioso en cuanto a la calidad del servicio educativo que están percibiendo los estudiantes, pues sin duda esto generará en un mediano plazo la satisfacción positiva para aquellos usuarios de este servicio, logrando en ellos 
fidelización y un mayor posicionamiento, tanto de la Institución como de la Facultad.

\section{Discusión}

Teniendo en cuenta la tabla 7, específicamente la parte relacionada con la interpretación de los resultados obtenidos con la aplicación del Modelo Servqual y las acciones que deben tomar con el fin de reducir las brechas entre expectativas y percepciones, es preciso mencionar a Ravichandran et al (2010) al exponer que, tanto académicos como comercializadores de servicios requieren maneras más efectivas y eficientes para proporcionar a los consumidores lo que ellos necesitan, ya que esto redundará en mejores niveles de satisfacción, fidelización y competitividad.

Siendo el Modelo Servqual una escala de medición que permite valorar las diferencias presentes entre las expectativas y las percepciones de los usuarios utilizando el diferencial semántico como parámetro de medición, es notorio que su uso en diversos campos en donde el servicio es una constante, es total- mente apropiado, pues conlleva a que se determine con precisión cuáles son las brechas existentes entre aquello que un cliente espera y lo que realmente percibe de lo recibido. Siendo así, es importante resaltar que la aplicación de este Modelo unido a un tipo de investigación Concluyente-Descriptivo, logra establecer dentro de los resultados arrojados cifras contundentes que, al ser interpretadas desde el Marketing, son el punto de partida para la toma de decisiones administrativas.

Para el presente caso, el haber implementado el tipo de investigación y el modelo de medición del servicio mencionados anteriormente, en una Institución de Educación Superior de la ciudad de Pereira en Colombia, mostró las deficiencias presentes en su oferta y que no están permitiendo que los estudiantes posean óptimos niveles de percepción; además, de que la institución entregue un servicio donde las dimensiones evaluadas se encuentren por encima del umbral de satisfacción esperado y en el cuadrante óptimo de servicio.

Tabla 7 Acciones a tomar de acuerdo a resultados

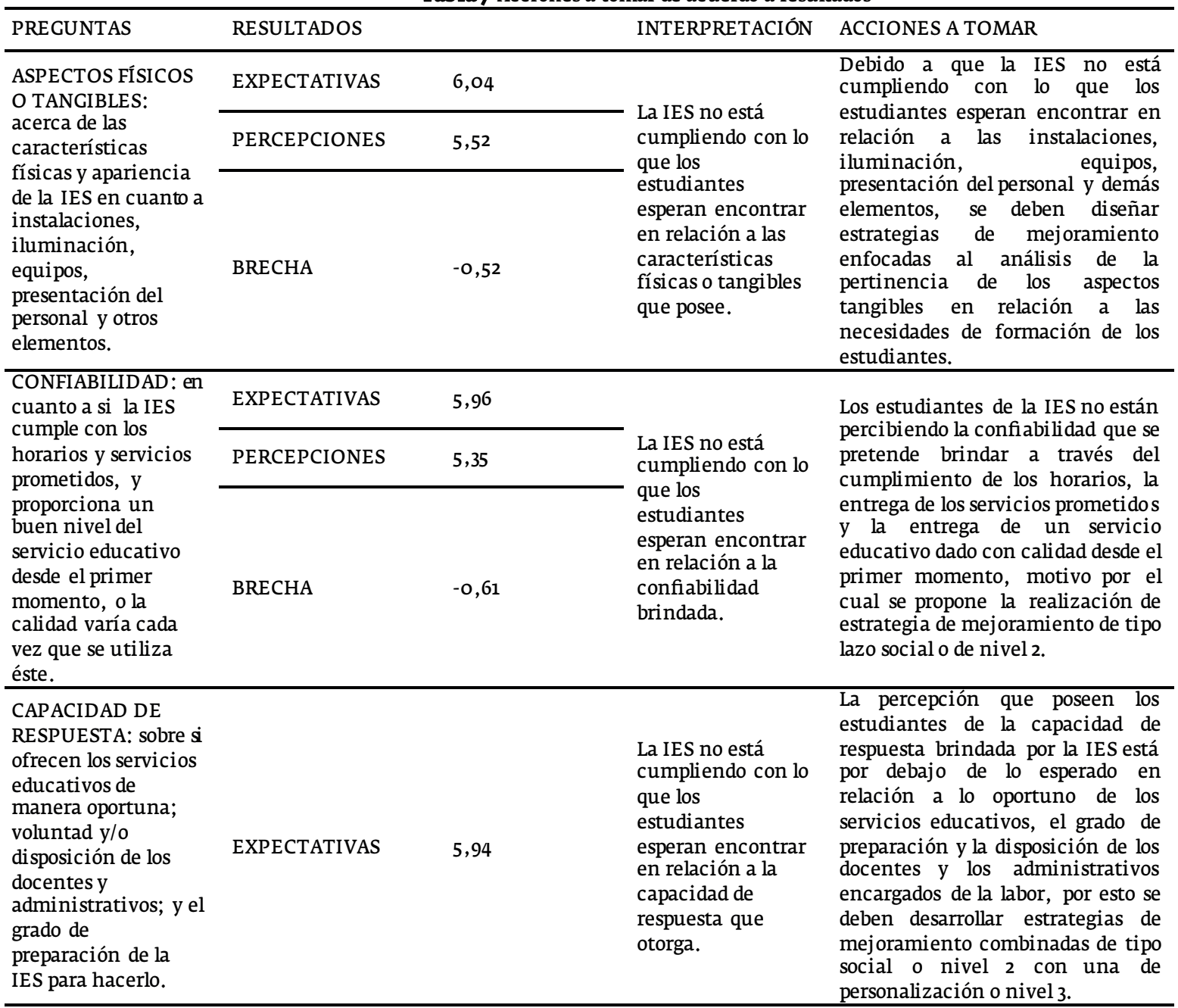




\begin{tabular}{|c|c|c|c|c|}
\hline $\begin{array}{l}\text { GARANTÍA: acerca } \\
\text { de la amabilidad que }\end{array}$ & EXPECTATIVAS & 6,20 & \multirow{3}{*}{$\begin{array}{l}\text { La IES no está } \\
\text { cumpliendo con lo } \\
\text { que los } \\
\text { estudiantes } \\
\text { esperan encontrar } \\
\text { en relación a la } \\
\text { garantía y } \\
\text { seguridad } \\
\text { brindadas. }\end{array}$} & \multirow{3}{*}{$\begin{array}{l}\text { La percepción de la garantía dada } \\
\text { en términos de la amabilidad del } \\
\text { personal en la atención, en la } \\
\text { confianza, credibilidad y } \\
\text { protección frente a las operaciones } \\
\text { que se realizan es negativa, } \\
\text { aspecto que implica la } \\
\text { implementación de estrategias de } \\
\text { mejoramiento combinadas de tipo } \\
\text { socialo nivel 2, de personalización } \\
\text { o nivel } 3 \text { y una de lazos } \\
\text { estructurales o de nivel } 4 \text {. }\end{array}$} \\
\hline $\begin{array}{l}\text { el personal de la } \\
\text { institución ofrece a } \\
\text { sus estudiantes y la }\end{array}$ & PERCEPCIONES & 5,63 & & \\
\hline $\begin{array}{l}\text { seguridad de sus } \\
\text { operaciones. } \\
\text { Confianza, } \\
\text { credibilidad y } \\
\text { protección frente a } \\
\text { los servicios } \\
\text { educativos que } \\
\text { ofrece la IES a sus }\end{array}$ & BRECHA & $-0,58$ & & \\
\hline
\end{tabular}

EMPATIA: en cuanto

a si en la IES

experimentan los

sentimientos de

otros como propios.

Entienden las

necesidades de sus

estudiantes y

colocan los servicios

educativos a su

alcance; la atención

personalizada se da

cuando es solicitada

y se ofrecen los

horarios de atención

convenientes.

$\begin{array}{cl}\text { EXPECTATIVAS } \quad 6,01 \quad & \begin{array}{l}\text { La IES no está } \\ \text { cumpliendo con lo } \\ \text { que los } \\ \text { estudiantes } \\ \text { esperan encontrar } \\ \text { en relación a la } \\ \text { empatía ofrecida. }\end{array}\end{array}$

La dimensión de empatía ha generado una percepción negativa frente a lo esperado; ésta dimensión se relaciona con los sentimientos experimentados en sentimientos experimentados en
los estudiantes por el entendimiento de sus necesidades a través de los servicios recibidos, motivo por el cual, la IES debe desarrollar estrategias de mejoramiento combinadas entre una de tipo social o nivel 2 y una personalizada o de nivel 3 .

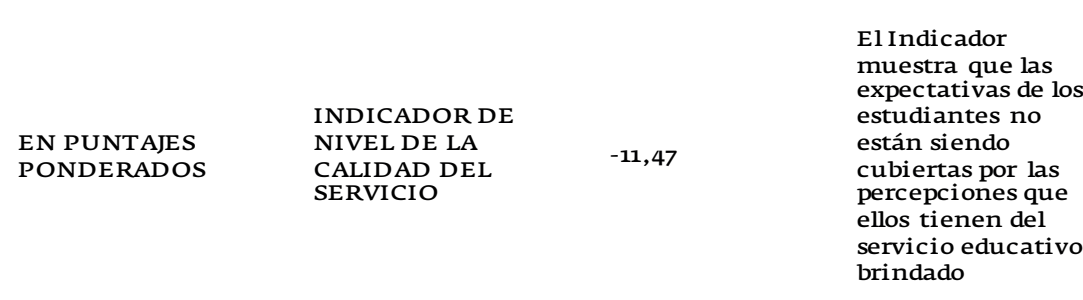

TIEMPO PERDIDO NINGUNA

Los estudiantes

reconocen como

fortalezas de la

institución las

dimensiones

relacionadas con

ASPECTOS

FISICOS O

DISGUSTOS

EMPATÍA

EN EL

POSICIONAMIENTO

DE LAS

DIMENSIONES EN

LOS CUATRO

CUADRANTES

OPORTUNIDADES NINGUNA
DE MEJORA

la Confiabilidad,

la Garantía y la

Capacidad de

Las dimensiones

de Aspectos

Tangibles y

Empatía se

encuentran en el

cuadrante de

Disgustos

Menores, es decir,

que los

estudiantes

reclaman unas

CONFIABILIDAD
FORTALEZAS

/ GARANTÍA/

CAPACIDAD DE

RESPUESTA

condiciones en las

instalaciones y

locaciones de la

institución, así

como un mayor

entendimiento a

sus necesidades.

\begin{tabular}{|c|c|c|c|}
\hline $\begin{array}{l}\text { EN EL } \\
\text { POSICIONAMIENTO }\end{array}$ & $\begin{array}{l}\text { EXPECTATIVAS } \\
\text { SATISFECHAS }\end{array}$ & $\begin{array}{l}\text { ASPECTOS } \\
\text { FÍSICOS O } \\
\text { TANGIBLES / } \\
\text { EMPATÍA } \\
\text { GARANTÍA/ } \\
\text { CAPACIDAD DE } \\
\text { RESPUESTA } \\
\end{array}$ & $\begin{array}{l}\text { Cuatro de las cinco } \\
\text { dimensiones de la } \\
\text { calidad del servicio } \\
\text { educativo }\end{array}$ \\
\hline DIMENSIONES EN & & & estudiantes ser \\
\hline $\begin{array}{l}\text { EL UMBRAL DE } \\
\text { SATISFACCIÓN }\end{array}$ & $\begin{array}{l}\text { OPORTUNIDADES } \\
\text { DE MEJORA }\end{array}$ & CONFIABILIDAD & $\begin{array}{l}\text { hallan por encima } \\
\text { del umbral de } \\
\text { satisfacción y una } \\
\text { por debajo de éste. }\end{array}$ \\
\hline
\end{tabular}

La ponderación negativa de la evaluación de las percepciones en relación a las expectativas frente al servicio entregado por la IES, permite corroborar los resultados adversos obtenidos en la evaluación individual de cada dimensión, dado a lo anterior, se establece que se deben desarrolla $r$ establece que se deben desarrolla r
estrategias combinadas de análisis de pertinencia de las instalaciones, de tipo lazos sociales 0 nivel 2 , de tipo personalizada o nivel 3 y de tipo lazos estructurales o nivel 4.

No aplica

Al encontrarse esta dos dimensiones (aspectos tangibles y empatía) en el cuadrante de disgustos, hacen que la implementación de estrategias enfocadas a ellas (análisis de pertinencia de las instalaciones, de tipo lazo social $y$ de tipo personalización), implementarse con prioridad, ya que son los aspectos a mejorar rápidamente.

No aplica

Según el posicionamiento de las dimensiones en los cuadrantes, se destaca que la confiabilidad, la garantía y la capacidad de respuesta se ubican como fortalezas, motivo por el cual se proponen estrategias de sostenimiento combinadas de nivel 3 o tipo personalización, campañas de difusión de los servicios y programas de servicios y programas de
formación al personal encargado de los procesos de prestación.

Se deben implementar de manera prioritaria oportunidades de mejora que permitan acercar las percepciones a las expectativas. Es de resaltar que a pesar de que cuatro de las cinco dimensiones están por encima del umbral, las brechas negativas y la cercanía con el umbral hace que sea urgente tomar medidas. Debido a lo estrategias combinadas de análisis estrategias combinadas de análisis tipo lazo social, de personalización y de tipo lazo estructural. 
La investigación ha mostrado la aplicación que Parasuraman et al (1985) proponen con el Modelo Servqual y sus cinco dimensiones del servicio; sin embargo, es válido mencionar que de acuerdo al sector o actividad en la que esta herramienta de medición de la calidad del servicio vaya a ser utilizada, es preciso conocerlo de manera detallada para poder elaborar los ajustes o la adaptación que las dimensiones y los cuestionarios requieran, pues de esta manera existirá una mayor posibilidad de éxito en los resultados con el fin de implementar las estrategias necesarias para reducir al máximo las brechas existentes entre las expectativas de un servicio y su percepción al recibirlo.

\section{Conclusiones}

1. La utilización del Modelo Servqual permitió efectuar la medición de las cinco dimensiones propuestas por Parasuraman (aspectos físicos o tangibles, confiabilidad, capacidad de respuesta, garantía y empatía), y en cuyos resultados se observan brechas negativas en cada una de las dimensiones evaluadas, con lo que se da respuesta al objetivo propuesto de determinar las brechas existentes entre las expectativas y las percepciones de la calidad del servicio educativo por parte de los estudiantes de una Facultad de Ciencias Administrativas en una IES privada de la ciudad de Pereira. 2. El indicador de nivel de calidad del servicio, y el posicionamiento de las dimensiones con su respectivo umbral de satisfacción, generan en las directivas de la institución una perspectiva más amplia de la situación de calidad que afronta el servicio, lo que a su vez permite la generación y la posibilidad de toma de decisiones estratégicas con focalización táctica. 3. Procesos en los cuales investigaciones como la contenida en el presente artículo con la aplicación del Modelo Servqual, demuestran la utilidad de su uso con miras al mejoramiento continuo del servicio educativo con parámetros de calidad, pueden ser tenidos en cuenta en el seguimiento y cumplimiento de los lineamientos emitidos por los organismos de gobierno nacionales. Siendo así, es posible contribuir a la calidad de la educación en la medida que las IES implementen en sus procesos de autoevaluación y autorregulación la aplicación de este tipo de mediciones.

\section{Referencias}

ASCUN, A. (2014). Misión y Visión de ASCUN. Obtenido de http://www.sgc-ascun.org.co/index.php

Bryman, A. (2012). Social Research Methods. Oxford University Press (Oxford Uni, Vol. 4th editio). Oxford: OXFORD UNIV PRESS. Retrieved from file://C:/Users/ MacBook/Downloads/SocialResearchMethods.pdf

Cervates Collado, T., \& Jiménez Hernández, R. (2012). Calidad de servicio que proporcionan las Insticiones de Educación Superior en México. Obtenido de http:// search.proquest.com/openview//77a62a2889eza6ro1a8e- $\mathrm{d}_{3} 8 \mathrm{c} 36 \mathrm{~b} 2 \mathrm{fc} 2 \mathrm{o} / \mathrm{l}$ ? pq-origsite $=\mathrm{gscholar}$ Chaparro Pinzón, C. R., \& Duque Oliva, E. J. (2012). Medición de la percepción de la calidad del servicio de educación por parte de los estudiantes ce la UPTC Duitama. Criterio Libre, 159-192. Obtenido de https://search. proquest. com/docview/1024426730? accountid $=48797$

Cheruityot, T., \& Maru, L. (2013). Service quality and relative performance of public universities in East Africa. The TQM Journal, 25(5), 533-546. doi:10.1108/TQM-11-2012-0103 Congreso de Colombia. (1992). Ley 30 de diciembre 28 de 1992. Obtenido de http://www.oei. es/quipu/colombia/Ley educ superior.pdf

Giraldo G, U., Abad A, D., \& Díaz P, E. (s.f.). Bases para una política de calidad de la Educación Superior en Colombia. Obtenido de https://www.cna. gov.co/1741/articles-186502_doc_academico1o.pdf

Grönroos, C. (1984). A service quality model and its marketing implications. European Journal of Marketing, 18(4), 36-44. Hernández Martínez, J. (2008). La calidad en el servicio para la satisfacción del cliente. Revista Unisangil Empresarial, 25-30.

Hernández Sampieri, R., Fernández Collado, C. \& Baptista Lucio, P. (2014). Metodología de la Investigación. México D.F.: McGraw-Hill / Interamericana Editores, S.A.

Kassian Rank, A. A., Godinez Cubillo, N., \& Corral Kassian, E. (2014). Calidad en el Servicio. Obtenido de Clinica del Dolor: http://www.ametd.org/web/images/revista/Algos $\% 202014 / A L G O S \% 20$ No $\% 206-2014$. pdf $\#$ page $=6$

Kotler, P., \& Keller, K. (2014). Dirección de Marketing. México D.F.: Pearson Educación.

Lewis, B., \& Mitchel, V. (1990). Defining and measuring the quality of customer service. Marketing Intelligence \& Planning , 8(6), 11-17.

Lorenzatti, M. (s.f.). Marketing para Insticiones y Centros de Educación contínua. Obtenido de https://docs.google.com/file/d/oB-p5qHi6ZI8DX1dkNW1FcGFoQUo/edit

Lovelock, C. , \&Wirtz, J. (2009). MarketingdeServicios. Personal, tecnología y estrategia. México: Pearson Educación. Malhotra, N. (2008). Investigación de Mercados. México D.F.: Pearson Educación.

Martínez, Sergio (2012). Publicado en Gestión de Servicios de TI. Recuperado de: https://es.scribd.com/ document/115652966/Servqual-calidad-del-servicio

Ministerio de Educación Nacional - República de Colombia. (2006). Plan Decenal de Educación debate público 2006 2016. Obtenidodehttp://www.plandecenal.edu.co/cms/ media/herramientas/pnde_2006_2016_compendio.pdf

Ministerio de Educación Nacional - República de Colombia. (14 de Junio de 2010). Instiruciones de Educación Superior. Obtenido de https://www.mineducacion. gov.co/1759/w3-article-231240.html

Ministerio de Educación Nacional - República de Colombia. (2010). Sistema de Educación Superior. Obtenido de http://www.mineducacion.gov.co/1621/w3-article-235585. html

Ministerio de Educación Superior - República de Colombia. (2013). Misión y Visión de la Educación . Obtenido de http://www.mineducacion.gov.co/1621/w3-article-89266.html

Naranjo Hernández, C. (2011). Marketing educativo; desarrollo de una estrategia C.E.M. aplicado a la Universidad nacional de Colombia sede Manizales como base para la fidelización de clientes y complemento a la estrategia de C.R.M. Obtenido de http://bdigital.unal. edu.co/3837/1/catalinanaranjohernandez.2011.pdf

Ospina Díaz, M., \& Sanabria Rangel, P. (2010). Un enfoque de mercadeo de servicios educativos para la gestión de las organizaciones de educación superior en colombia: el modelo MICME. Revista Facultad de Ciencias Económicas: Investigación y reflexión, 107-136.

Parasuraman, A., Zeithaml, V. A., \& Berry, L. L. (1985). A conceptual model of service quality and its implications for future research. Journal of Marketing, 49, 41-50.

Parasuraman, A., Zeithaml, V., \& Berry, L. (1985b). Alternative scales for measuring service quality: a compartive assessment based on psychometrics test and diagnostic criteria. Journal of Retailing, 70(3), 201-230.

Parasuraman, A., Zeithaml, V., \& Berry, L. (1988). Servqual: a multiple-item scale for measuring consumer percep- 
tions of service quality. Journal of Retailing, 64(1), 12-40.

Pérez Juste, R. (2005). Calidad de la educación, calidad en la educación. Hacia su necesaria integración. Educación XXI, 11-33.

Pérez Solorzano , C. (2010). Modelo de estandarización de cadenas de servicios personales en base a la medición de la satisfacción del cliente . Obtenido de http:// www.dspace.espol.edu.ec/bitstream/123456789/11101/3/ Modelo $\%$ ode $\% 20$ estandarizaci $\%$ C 3\% B 3n $\% 20$ de $\% 20$ cadenas $\% 2$ ode $\% 2$ oservicios $\% 2$ opersonale.pdf

Pineda, U., Estrada, M., \& Parra, C. (2011). Aplicación del modelo Servqual y herramientas de ingeniería de la calidad para la planificación del servicio en la Biblioteca Central de la Universidad de Antioquia. Revista Interamericana de Bibliotecología, 243-255.

Ravichandran, K., et al (2010). Influence of Service Quality on Customer Satisfaction Application of Servqual Model. International Journal of Business and Management. 5(4) 117-124

Redacción M\&M. (18 de Diciembre de 2008). Clientes in satisfechos: iproblema u oportunidad? . Obtenido de http://musicaymercado.org/clientes-insatisfechos/

Ríos, J., \& Santomá, R. (2008). Calidad de servicio en la Industria Hotelera desde la perspecti- va del SERVQUAL. Management Empresa, 1-12. Rodríguez Ponce, E., Arenada Guirriman, C., \& Pedraja Rejas, L. (2011). El impacto de la calidad del servicio sobre la calidad institucional en las universidades: evidencia empírica desde Chile. Revista de Ciencia y Tecnología de América, 657-663.

Schendel, D., \& Hatten, K. (Septiembre de 1972). Business policy and strategic management. A broader view for an emerging discipline. Obtenido de https://files.eric.ed.gov/fulltext/ED102732.pdf Thiétart, R.-A. et al. (1999). Doing management research. In M. Girod-Séville \& V. Perret (Eds.), Doing Management Research: A comprehensive guide. (British Li, pp. 1-30). London: SAGE publications Ltd. Torres Moraga, E., \& Araya Castillo, L. (2010). Construcción de una escala para medir la calidad del servicio de las universidades: Una Aplicación al Contexto Chileno. Revista de Cinecias Sociales, 54-67. Zeithaml, V., Bitner, M. J., \& Gremler, D. (2009). Marketing de Servicios. México: McCraw Hill. 